\title{
Cuerpo extraño en fosas nasales y conducto auditivo externo en el Hospital de Clínicas
}

\author{
Foreign body in nostrils and ear canal at Hospital de Clinicas

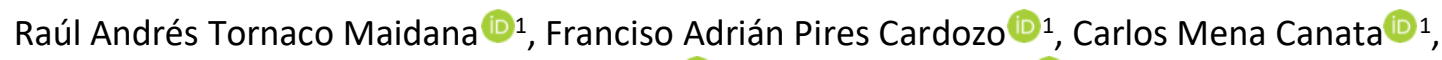 \\ Enrique Pérez Girala ${ }^{1}$, César Franco Peña ${ }^{1}$.
}

${ }^{1}$ Univerisdad Nacional de Asunción, Facultad de Ciencias Médicas, San Lorenzo, Paraguay.

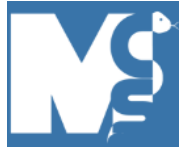

Recibido: 03/09/2021

Revisado: 01/12/2021

Aceptado:04/01/2022

\section{Autor correspondiente}

Raúl Andrés Tornaco Maidana Universidad Nacional de Asunción, Paraguay

raultornaco@gmail.com

\section{Conflictos de interés}

Los autores declaran no poseer conflictos de interés.

\section{Fuente de financiación}

Los autores no recibieron apoyo financiero del Gobierno Regional de Tumbes para realizar esta investigación.

Este artículo es publicado bajo una licencia de Creative Commons Reconocimiento 4.0 Internacional.

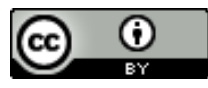

\section{RESUMEN}

Introducción: La presencia de cuerpo extraño en cavidad nasal u ótica es un problema frecuente en pediatría, porque puede producir secuelas severas para la salud del paciente. Estratificar por edades nos ayudaría a definir las medidas más apropiadas ente estos eventos en las distintas edades. Objetivo: Conocer la diferencia de media de edades entre pacientes con cuerpo extraño ótico y nasal. Metodología: Estudio observacional descriptivo con componentes analíticos, de muestreo aleatorio simple. De pacientes pediátricos del Hospital de Clínicas con antecedentes de introducción de cuerpo extraño en fosas nasales y conducto auditivo externo de enero a junio del 2019. Resultados: Fueron analizadas 182 interconsultas al servicio de otorrinolaringología, de pacientes pediátricos con antecedente de cuerpo extraño en oído o fosa nasal. El 47,81 \% era del sexo femenino. En el 58,79 \% de los casos el motivo de consulta era cuerpo extraño en fosa nasal y en el $41,21 \%$ en oído. Los métodos de extracción más frecuentes fueron la cureta nasal $(87,85 \%)$ y el lavado de oído (54,67\%). Los cuerpos extraños más frecuente fue piezas de bijouteri. Solo 2 $(1,09 \%)$ pacientes requirieron internación (ambos con retraso en el desarrollo sicomotor) y extracción bajo sedación. El tratamiento más usado posterior a la extracción de oído fue antibióticos tópicos (52 \%) y; antibióticos orales combinados con lavados nasales $(28,97 \%)$ para los de fosa nasal. La media de edades de pacientes con cuerpo extraño en fosa nasal fue de 2,97 años y en oído 5,59 años, significativamente mayor $(p<0,05)$. Conclusión: Las guías refieren que los niños a partir de los 5 años distinguen lo bueno y lo malo, disminuyen las autointroducciones y esto se vuelve algo más bien accidental; sin embargo, en nuestra experiencia la edad promedio de introducción oído era mayor a 5 años.

Palabras clave: Cuerpo extraño; Cavidad Nasal; Conducto auditivo externo; Extracción.

\section{ABSTRACT}

Introduction: The presence of a foreign body in the nasal or otic cavity is a frequent problem in pediatrics, because it can produce severe sequels for the patient's health. Stratifying by age would help us to define the most appropriate measures between these events at different ages. Objective: To know the average age difference between patients with otic and nasal foreign bodies. Methods: Descriptive observational study with analytical components, simple random sampling. Pediatric patients at the Hospital de Clínicas with a history of introduction of foreign body into the nostrils and external auditory canal from January to June 2019. Results: 182 interconsultations were analyzed in the otolaryngology service of pediatric patients with a history of foreign body in the ear or nostril. $47.81 \%$ were female. In $58.79 \%$ of the cases the reason for consultation was foreign body in the nostril and in $41.21 \%$ in the ear. The most frequent extraction methods were nasal curettage (87.85\%) and earwashing (54.67\%). The most frequent foreign bodies were pieces of bijouteri. Only 2 (1.09\%) patients required hospitalization (both with delayed psychomotor development) and extraction under sedation. The most commonly used treatment after ear removal was topical antibiotics (52\%) and oral antibiotics combined with nasal lavage $(28.97 \%)$ for nasal fossa. The mean age of patients with foreign body in the nasal fossa was 2.97 years and in the ear 5.59 years $(p<0.05)$. Conclusion: The guidelines state that children from 5 years onwards distinguish good and bad, decrease autointroductions and this becomes rather accidental; however, in our experience the average age of ear introduction was greater than 5 years.

Keywords: Foreign Body; Nostril; Ear; Removal. 


\section{INTRODUCCIÓN}

La presencia de cuerpo extraño en cavidad nasal u ótica es un problema muy frecuente en la población pediátrica, en todos los niveles socioeconómicos, incluso en países desarrollados de Europa, hasta el $2 \%$ de la población pediátrica acude a consulta por esta causa (1). Un cuerpo extraño en fosa nasal puede producir secuelas desde sobreinfección hasta paso a vías respiratorias bajas o constituir una verdadera urgencia (2), en cuanto a los que se encuentran en el conducto auditivo, otitis externa crónica, perforación timpánica, entre otras complicaciones (3).

No existe estadísticas en nuestro medio sobre la prevalencia de este fenómeno, pero no hay ninguna duda de que es un frecuente motivo de interconsultas del servicio de pediatría al otorrinolaringólogo; hasta el $50 \%$ de las consultas pediátricas se relacionan a presencia de cuerpos extraños en cavidades y es un verdadero desafío para los profesionales el cómo encarar a dicha situación, debido a las múltiples presentaciones, distintos objetos, tiempo de evolución, posibilidad de complicaciones, y de la poca colaboración de los pacientes pediátricos en general (4). Estratificar por rango etario nos ayudaría a definir cuáles son las medidas de precaución más apropiadas para estos eventos en las distintas poblaciones. El objetivo de este trabajo es dar a conocer la diferencia de media de edades entre pacientes con cuerpo extraño (CE) ótico y nasal en el Hospital de Clínicas en el año 2019 (5).

\section{METOdOLOGÍA}

El diseño de estudio fue descriptivo de corte transversal y temporalmente retrospectivo con muestreo probabilístico aleatorio simple. La población enfocada constituida por pacientes pediátricos con antecedentes de introducción de cuerpo extraño en cavidad nasal y conducto auditivo externo. Habiendo accedido a pacientes pediátricos con antecedentes de introducción de cuerpo extraño en cavidad nasal y conducto auditivo externo, que han acudido a la Urgencia Pediátrica del Hospital de Clínicas, San Lorenzo, en el periodo Enero 2018-Marzo 2019.

En cuanto a criterios de inclusión hemos tenido en consideración pacientes con cuerpo extraño nasal u ótico de 0 a 18 años, que habían recurrido al servicio de urgencias pediátricas. Habiéndose excluido a los pacientes con fichas incompletas o mal redactadas. En cuanto al tamaño muestral, se ha realizado un pre test en el cual se determinó que en una semana el $30 \%$ de las interconsultas a pediatría, a otorrinolaringología, constituían pacientes con diagnósticos. Según el cálculo de tamaño muestral se ha obtenido una " $n$ " mínima de 164 pacientes (3), utilizándose un intervalo de confianza del $95 \%$. Las variables estudiadas fueron sexo (hombre o mujer), procedencia, localización del cuerpo extraño (fosa nasal derecha, fosa nasal izquierda, ambas fosas nasales, oído derecho, oído izquierdo, ambos oídos), método de extracción en nariz (cureta, aspirado, no se ha constatado), método de extracción en oídos (lavados, hartman, aspirado, cureta de oído, no se ha constatado), tipo de cuerpo extraño extraído, internación (si o no), complicación en cuerpos extraños en fosas nasales (fetidez y rinorrea purulenta, epistaxis, fiebre, ninguna), complicaciones en cuerpos extraños en oídos (dolor, sangrado, pus, fiebre, ninguna), tratamiento posterior a extracción, edad (en años cumplidos), tiempo de evolución (en días)

Los datos han sido analizados de la siguiente manera, se han recolectado utilizando las fichas del servicio de urgencias pediátricas del Hospital de Clínicas, habiéndose almacenados en Excel 2010 y analizados con el sistema Epi-Info versión 7.2.2.6. Se procesaron los datos mencionados con medidas de tendencia central para las variables cualitativas, y prueba de $t$ de Student para los análisis de datos. Desde el punto de vista ético, este estudio no ha representado riesgo para la integridad física y psíquica del paciente. Los datos que se han recabado de las fichas no incluyeron datos que hayan podido descubrir la identidad del paciente. Se ha respetado el principio de: Beneficencia: En caso de resultados de alarma se ha proporcionado información al sujeto, para acudir a ayuda profesional. No maleficencia: Los datos obtenidos en esta encuesta no han sido utilizados para otros fines ajenos a la investigación en este estudio y presentación de casos. Justicia: Todos los pacientes han tenido la misma oportunidad de participar en la encuesta. Autonomía: Cualquiera de los participantes podía haberse negado a participar del estudio sin haber existido coacción para lo contrario.

\section{RESULTADOS}

Se analizaron 182 fichas de interconsultas de pacientes pediátricos con diagnóstico de cuerpo extraño en oído (CIE10: T16) y en fosa nasal (T171). En cuanto a la distribución por sexo el $48 \%$ fue femenino, y el $52 \%$ masculino. La media de edades es 4,22 años con un desvío estándar de $\pm 2,83$ años. La diferencia de las medias de los grupos de pacientes con cuerpo extraño en fosa nasal y conducto auditivo externo, según prueba $t$ para dos muestras suponiendo varianzas desiguales, se detalla en la Tabla 1 , con diferencias significativas entre las medias de edades, siendo mayor para el grupo con cuerpo extraño en conducto auditivo externo $(p<0,05)$. 
TABLA 1: MEDIA DE EDADES, FCM - UNA CÁTEDRA Y SERVICIO DE OTORRINOLARINGOLOGÍA 2019 (N=182)

\begin{tabular}{lll}
\hline Edades & Fosa Nasal & Oído \\
\hline Media & 2,97 & 5,59 \\
Varianza & 2,15 & 9,91 \\
\hline
\end{tabular}

El 58,79\% de los pacientes presentó diagnóstico de cuerpo extraño en fosa nasal, de los cuales 51,4 \% estaban en la fosa nasal derecha y el $46,72 \%$ en la izquierda, en $1,86 \%$ se encontraba en ambas fosas nasales. El método de extracción fue en el $88 \%$ de los casos con cureta, en el $10 \%$ no se constata y en el $2 \%$ aspirado. El $41,2 \%$ se encontraba en el conducto auditivo, de estos 56 \% estaban en derecho, 38,67\% estaban en el izquierdo, y el 5,3\% en ambos oídos. El método de extracción fue en el $56 \%$ de los casos lavado, $25 \%$ Hartman, $7 \%$ aspirado, $7 \%$ no se constata y el $5 \%$ cureta de oído.

En cuanto a los cuerpos extraños que más se extrajeron fueron piezas de bisutería en el $21,42 \%$, semillas en el $19,23 \%$, insectos en el $8,24 \%$ y papel en el $7,14 \%$. En total el 53,5 \% de los cuerpos extraños hallados eran orgánicos y el $46,5 \%$ de los objetos eran inorgánicos. El tiempo de evolución en promedio fue de 2,32 días, con un rango de media hora a 90 días siendo para cuerpos extraños en fosa nasal de 3,25 días y 1,25 días para los que se encontraban en oído. En el 13,18 \% de los pacientes no saben precisar el tiempo de evolución.

En el 87,9 \% de los pacientes se tenía antecedentes de autointroducción del cuerpo extraño. En 165 (90,65 \%) de los casos se constató un solo objeto, en 6 casos $(3,29$ $\%)$ se encontró más de un objeto, en 11 casos (6,04\%) no se encontró ningún cuerpo extraño. En 2 pacientes $(1,09 \%)$ se requirió internación debido a la dificultad para la extracción del cuerpo extraño. En la Tabla 2 se pueden ver las complicaciones más frecuentes.

El tratamiento más usado posterior a la extracción de cuerpo extraño en conducto auditivo fue antibióticos tópicos (52 \%) y; antibióticos por vía oral combinados con lavados nasales $(28,97 \%)$ para los hallados en fosa nasal.

\section{TABLA 2: COMPLICACIONES DE CUERPOS EXTRAÑOS EN NARIZ Y OÍDO, FCM - UNA CÁTEDRA Y SERVICIO DE} OTORRINOLARINGOLOGÍA 2019 (N=182).

\begin{tabular}{lll}
\hline Complicaciones & Frecuencia & Porcentaje \\
\hline Nariz ( $\mathrm{n}=112$ ) & & \\
Fetidez y rinorrea purulenta: & 27 & 25,23 \\
Epistaxis & 7 & 6,54 \\
Fiebre & 3 & 2,8 \\
Ninguna & 75 & 70,09 \\
Oído ( $\mathrm{n}=70)$ & & \\
Dolor & 6 & 8 \\
Sangrado & 3 & 4 \\
Pus & 2 & 2,67 \\
Fiebre & 1 & 1,33 \\
Ninguna & 58 & 77,33 \\
\hline
\end{tabular}

\section{DISCUSIÓN}

En nuestra experiencia, los cuerpos extraños en oído y nariz suponen un motivo de consulta frecuente en los servicios de urgencias de pediatría, así como de interconsultas con otorrinolaringología de nuestro hospital, como también se informa en un estudio del departamento de ORL del Queen Elizabeth University Hospital, Glasgow, UK (3). El sitio más frecuente de alojamiento de cuerpo extraño en población pediátrica en un estudio realizado en la Universidad de Ciencias Médicas de Matanzas, Cuba fue el oído y en segundo lugar la cavidad nasal, sin embargo, en nuestro estudio el sitio mas frecuente fue la fosa nasal $(6,7)$.
Los cuerpos extraños en cavidades nasales, tienen el agregado de que pueden producir infección local en el sitio, como además ser aspirados hacia vías respiratorias bajas, y causar incluso hasta el $7 \%$ de mortalidad, situación que en nuestro estudio no se dio como complicación (8).

Las guías consultadas sugieren que estos pacientes sean derivados a los servicios de otorrinolaringología en casos de imposibilitarse la extracción o antecedentes de otorrea $u$ otorralgia, y atresia de coanas, epistaxis importante o neoformación en fosa nasal, sin embargo, en nuestro hospital, ante el mero antecedente de presencia del cuerpo extraño en cavidad nasal o conducto auditivo es motivo de 
interconsulta al servicio de otorrinolaringología (9-11).

La guía de manejo de cuerpos extraños en oído y nariz del Hospital Son Dureta de Mallorca, refiere que los niños a partir de los 5 años distinguen que es bueno o malo para ellos mismos, disminuyendo las autointroducciones y se producen estos eventos, más bien accidentalmente; sin embargo, en nuestra experiencia, la edad promedio de introducción de CE en CAE era mayor a 5 años $(4,9,10)$.

En cuanto a la extracción de CE de fosas nasales, utilizamos lo recomendado en dicha guía (gancho de punta roma, en nuestro caso, artesanal, utilizamos el mango del espejo laríngeo) en la mayor cantidad de los casos, sin embargo, en oído, los métodos utilizados fueron más variados, siendo de más frecuencia el lavado. Los cuerpos extraños extraídos en su mayoría resultaron de origen orgánico (poco más de la mitad), en otros estudios se han reportan hasta el $91 \%$ orgánicos $(10,12,13)$.

La prevalencia de complicaciones fue baja, siendo solo del casi el $30 \%$ en fosa nasal y $22,33 \%$ en conducto auditivo, y ninguna de ellas de gravedad, coincidentemente con el estudio antes mencionado de Cuba, la Matanza, en el cual solo el $86 \%$ no ha presentado algún tipo de complicación $(6,14-19)$.

En nuestro estudio concluimos que existe una diferencia significativa, entre las medias de edades de pacientes con cuerpo extraño en cavidad nasal y conducto auditivo, resultando significativamente mayor para los que presentaban en oído, consideramos un dato de alto valor a la hora de definir qué medidas tomar para prevención y posteriormente tratamiento ante dicha situación, puesto que la colaboración y fuerza difiere según el grupo etario y las medidas de contención a las que se recurren son diferentes, y la manipulación sin una correcta técnica puede llevar a severas consecuencias $(11,17,18)$.

\section{CONTRIBUCIÓN DE LOS AUTORES}

TM-RA, PC-FA, MC-C, GP-E y FP-C han participado de la concepción y diseño del trabajo, recolección y obtención de resultados, análisis e interpretación de datos, redacción del manuscrito, revisión crítica del manuscrito y aprobación de su versión final.

\section{REFERENCIAS}

1. Schuldt T, Großmann W, Weiss NM, Ovari A, Mlynski R, Schraven S. Aural and nasal foreign bodies in children Epidemiology and correlation with hyperkinetic disorders, developmental disorders and congenital malformations. International Journal of Pediatric Otorhinolaryngology. 2019;118:165-169.

https://doi.org/10.1016/i.ijporl.2019.01.006

2. Jahshan F, Sela E, Gruber M. Pneumothorax and
Pneumomediastinum Complicating Pediatric Foreign Body Aspiration. Ear, Nose Throat J. 2020;99(2):107-108. https://doi.org/10.1177/0145561318824225

3. Woodley N, Mohd Slim MA, Tikka T, Locke RR. Not 'just' a foreign body in the ear canal. BMJ Case Rep. 2019;12(4): e229302. https://doi.org/10.1136/bcr-2019-229302

4. Artigas-Sapiain C, Sarría-Echegaray P, Tomás-Barberán M. Manejo de cuerpos extraños nasales y óticos en niños. Semer - Med Fam. 2010;36(5):278-282. https://doi.org/10.1016/j.semerg.2010.01.017

5. French MA, Lorenzoni G, Purnima, Azzolina D, Baldas, S, Gregori D, Gulati A. Foreign Body injuries in children in India: Recommendations for prevention from a comparative analysis with international experience. Int J Pediatr Otorhinolaryngol. 2019;124:6-13. https://doi.org/10.1016/j.ijporl.2019.05.020

6. Díaz Estévez LM, Naranjo Rodríguez SA, Negret Hernández M. Comportamiento de los accidentes por cuerpo extraño en Otorrinolaringología Pediátrica. RevMedElectrón. 2015;37(4):356-63. URL.

7. Torres Márquez M, Fonseca Pelegrín CL, Díaz Martínez MD, del Campo Mulet OA, Roché Hernández R. Accidentes en la infancia: una problemática actual en pediatría. MEDISAN. 2010;14(3):368-378. URL.

8. Rodríguez H, Cuestas G, Gregori D, Lorenzoni G, Tortosa S, Rodríguez D'Aquila, et al. Recomendaciones sobre la prevención de la aspiración de cuerpos extraños orgánicos. Arch Argent Pediatría. 2017;115(5):512-516. URL.

9. Cabrera García A, Quintana Luzón C. Cuerpos extraños en conducto auditivo externo y fosas nasales. JANO. 2001;1425:27-28. URL.

10. Lara C, Faba G, Caro J. Diagnóstico, manejo y actualización en cuerpo extraño aerodigestivo. Rev Otorrinolaringol Cir Cabeza Cuello. 2008;68(3):309-18. http://dx.doi.org/10.4067/S0718-48162008000400013

11. González-Hernández CB, Ríos-Nava JR, Curiel-Dávalos CB, Felipe-Vega JC, Vázquez-Ramírez JG. Complicaciones por manipulación previa de cuerpos extraños del oído y la nariz en pacientes pediátricos. An Orl Mex. 2016;61(1):25-34. URL.

12. Nazar R, Cabrera N, Martelo G, Machiavello G, Naser A. Cuerpo extraño nasosinusal inusual: presentación de 3 casos. Acta Otorrinolaringológica Española. 2014;(65):109113. https://doi.org/10.1016/j.otorri.2013.11.002

13. Fried M. Cuerpos extraños nasales. Manual MSD versión para profesionales. 2017. URL.

14. Artigas-Sapiain C, Sarría-Echegaray P, Tomás-Barderán M. Manejo de cuerpos extraños nasales y óticos en niños. Medicina de Familia. SEMERGEN. Elsevier. 2010;(36):278282. https://doi.org/10.1016/i.semerg.2010.01.017

15. Baranowski K, Aaraj MSA, Sinha V. Nasal Foreign Body. StatPearls. StatPearls Publishing; 2021. URL.

16. Tasche KK, Chang KE. Otolaryngologic Emergencies in the Primary Care Setting. Med. Clin. North Am. 2017;101(3):641-656.

http://dx.doi.org/10.1016/j.mcna.2016.12.009

17. Awad AH, ElTaher M. ENT Foreign Bodies: An Experience. Int Arch Otorhinolaryngol. 2018;22(2):146151. https://dx.doi.org/10.1055\%2Fs-0037-1603922

18. Bressler K, Shelton C. Ear Foreign-Body Removal: A Review of 98 Consecutive Cases. Laryngoscope 1993;103(4 Pt 1):367-370. https://doi.org/10.1002/lary.5541030401

19. Tee $T$, Nasserallah $M$. The art of removing nasal foreign bodies. Ncbi. 2017;(9):107-112. https://dx.doi.org/10.2147\%2FOAEM.S150503 